\title{
KNOW-CKD (KoreaN cohort study for Outcome in patients With Chronic Kidney Disease): design and methods
}

Kook-Hwan Oh ${ }^{1 \dagger}$, Sue Kyung Park ${ }^{2 \dagger}$, Hayne Cho Park ${ }^{1}$, Ho Jun Chin ${ }^{1}$, Dong Wan Chae ${ }^{1}$, Kyu Hun Choi ${ }^{3}$, Seung Hyeok Han ${ }^{3}$, Tae Hyun Yoo ${ }^{3}$, Kyubeck Lee ${ }^{4}$, Yong-Soo Kim ${ }^{5}$, Wookyung Chung ${ }^{6}$, Young-Hwan Hwang ${ }^{7}$, Soo Wan Kim ${ }^{8}$, Yeong Hoon Kim ${ }^{9}$, Sun Woo Kang ${ }^{9}$, Byung-Joo Park ${ }^{2}$, Joongyub Lee ${ }^{10}$, Curie Ahn ${ }^{1 *}$

and Representing KNOW-CKD Study Group

\begin{abstract}
Background: The progression and complications of chronic kidney disease should differ depending on the cause (C), glomerular filtration rate category (G), and albuminuria (A). The KNOW-CKD (KoreaN Cohort Study for Outcome in Patients With Chronic Kidney Disease), which is a prospective cohort study, enrolls subjects with chronic kidney disease stages 1 to 5 (predialysis).

Methods/Design: Nine nephrology centers in major university hospitals throughout Korea will enroll approximately 2,450 adults with chronic kidney disease over a 5-year period from 2011 to 2015. The participating individuals will be monitored for approximately 10 years until death or until end-stage renal disease occurs. The subjects will be classified into subgroups based on the following specific causes of chronic kidney disease: glomerulonephritis, diabetic nephropathy, hypertensive nephropathy, polycystic kidney disease, and others. The eligible subjects will be evaluated at baseline for socio-demographic information, detailed personal/family history, office BP, quality of life, and health behaviors. After enrollment in the study, thorough assessments, including laboratory tests, cardiac evaluation and radiologic imaging, will be performed according to the standardized protocol. The biospecimen samples will be collected regularly. A renal event is defined by $>50 \%$ decrease in estimated GFR (eGFR) from the baseline values, doubling of serum creatinine, or end-stage renal disease. The primary composite outcome consists of renal events, cardiovascular events, and death. As of September 2013, 1,470 adult chronic kidney disease subjects were enrolled in the study, including 543 subjects with glomerulonephritis, 317 with diabetic nephropathy, 294 with hypertensive nephropathy and 249 with polycystic kidney disease.
\end{abstract}

Discussion: As the first large-scale chronic kidney disease cohort study to be established and maintained longitudinally for up to 10 years, the KNOW-CKD will help to clarify the natural course, complication profiles, and risk factors of Asian populations with chronic kidney disease.

Trial registration: No. NCT01630486 at http://www.clinicaltrials.gov.

Keywords: KNOW-CKD, Chronic kidney disease, Cohort, Etiology, Progression, Complication, Natural course

\footnotetext{
* Correspondence: curie@snu.ac.kr

${ }^{\dagger}$ Equal contributors

'Department of Internal Medicine, Seoul National University, 101 Daehakro,

Chongno Gu, Seoul 110-744, Korea

Full list of author information is available at the end of the article
} 


\section{Background}

Chronic kidney disease (CKD) is a rapidly growing disease worldwide. The prevalence of CKD in Korea is estimated to be $13 \%$ in the adult population [1]. Approximately $5 \%$ of the Korean adult population is estimated to have decreased renal function with an estimated glomerular filtration rate (eGFR) less than $60 \mathrm{ml} / \mathrm{min}$ per $1.73 \mathrm{~m}^{2}$ [1]. CKD, which leads to dialysis or kidney transplantation, presents a major threat to cardiovascular (CV) events and all-cause mortality $[2,3]$. A patient with CKD is more likely to die of $\mathrm{CV}$ disease than to start renal replacement therapy.

Since 2002, a large number of epidemiologic studies, in addition to the general population, have demonstrated a significantly increased risk of adverse consequences in subjects with albuminuria, even in the presence of normal or mildly decreased GFR $[4,5]$. This finding warrants a broader view of the early stages of CKD within the nephrology population to determine the variables associated with CKD and the increased risks of death and adverse outcomes [6]. However, the mechanistic link between albuminuria and adverse consequences has not been established.

Kidney Disease: Improving Global Outcomes (KDIGO) 2012 Clinical Practice Guideline for the Evaluation and Management of CKD recommends a new classification of CKD, based on the cause, GFR category, and albuminuria (CGA) [7]. The characteristics of CKD differ significantly depending on the underlying disease or the location of the pathologic-anatomic abnormalities. Therefore, we hypothesized that the progression and complications of CKD should be different among the disease subgroups, which cause CKD. A new perspective in the viewpoint of the "CGA" paradigm is required to analyze and interpret the various parameters associated with CKD.

Nevertheless, recently established CKD cohort studies [8-11] are primarily based on the CKD population with low GFR (less than $60 \mathrm{ml} / \mathrm{min}$ per $1.73 \mathrm{~m}^{2}$ ). The above studies targeted the cardiovascular complications from subjects with moderate or severe renal impairment. In these studies, the early stages of renal injury are excluded from their target population and the specific causes of CKD are not considered. In contrast, a new CKD cohort study, including all of the stages of the predialysis CKD population and considering the specific causes of CKD, may lead us to gain insight into understanding the natural progression of CKD and the association between $\mathrm{CKD}$ and $\mathrm{CV}$ outcomes or mortality.

The KNOW-CKD (KoreaN Cohort Study for Outcome in Patients With Chronic Kidney Disease), funded by the Korea Centers for Disease Control and Prevention (KCDC), was established in 2011 by a group of nephrologists, epidemiologists, biostatisticians, and clinical pathologists in Korea.
The goals of the KNOW-CKD are as follows: 1) to establish an adult pre-dialysis CKD cohort covering the early stages of CKD, as well as the advanced stages of CKD, 2) to investigate the natural history of CKD, such as renal progression, death, and complications with respect to the CGA categories, 3) to evaluate the etiologic factors for renal progression and related complications, 4) to identify the mechanistic link between renal injury and adverse outcomes, and 5) to identify the genetic and molecular influences on renal progression and complications.

In the present paper, we report our study design and methods and results from study participants registered from 2011 to 2013.

\section{Methods/Design \\ Organization}

The KNOW-CKD is a patient-based cohort study. Nephrologists working in nine clinical centers in the major university-affiliated hospitals and epidemiologists, pathologists and biostatisticians in a research modulating center are participating in this study. The basic protocol of the study was approved by the ethical committee of each participating center including Institutional Review Boards of Seoul National University Hospital, Severance Hospital, Kangbuk Samsung Medical Center, Seoul St. Mary's Hospital, Gil Hospital, Eulji General Hospital, Chonnam National University Hospital and Pusan Paik Hospital. Among the nine clinical centers, six centers are located in the metropolitan city of Seoul, one in a satellite area of Seoul, Gyeonggi-do (a province), and two in the southern area of Korea (i.e., Chonnam-do and Busan). Throughout the national health care system, Korea has 44 tertiarycare general hospitals. The distribution of our participating clinical centers is parallel to the distribution of the major hospitals in Korea. The study is supervised by the CKD Advisory Committee, which comprises members from the KCDC and the Korean Society of Nephrology (KSN) (NCT01630486 at http://www.clinicaltrials.gov).

\section{Study population}

Each participating center will enroll approximately 250 consecutive individuals over a 5-year period from 2011 until 2015, totaling 2,450 adult patients with CKD who provide written informed consent. The participating individuals will be monitored for approximately 10 years until death or until ESRD occurs.

The KNOW-CKD will enroll ethnic Korean patients with CKD who range in age between 20 years and 75 years. The CKD stages from 1 to 5 (predialysis), based on the eGFR, is calculated using the four-variable Modification of Diet in Renal Disease (MDRD) equation as follows:

eGFR $\left(\mathrm{ml} / \mathrm{min}\right.$ per $\left.1.73 \mathrm{~m}^{2}\right)=175 \times$ [serum $\mathrm{Cr}$ $(\mathrm{mg} / \mathrm{dl})]^{-1.154} \times[\text { age }]^{-0.203} \times[0.742$ if female $] \times[1.212$ if black], using serum creatinine concentrations measured 
at a central laboratory and an assay traceable to the international reference material [12].

Excluded subjects are those who 1) are unable or unwilling to give written consent, 2) have previously received chronic dialysis or organ transplantation, 3) have heart failure (NYHA class 3 or 4) or liver cirrhosis (Child-Pugh class 2 or 3), 4) have a past or current history of malignancy, 5) are currently pregnant, or 6) have a single kidney due to trauma or kidney donation.

We defined and allocated the specific causes of the CKD into four subgroups: glomerulonephritis (GN), diabetic nephropathy (DN), hypertensive nephropathy (HTN), and polycystic kidney disease (PKD). The definition of the subgroup is defined by the pathologic diagnosis, in the event that the biopsy result is available. Otherwise, the subgroup classification depends on the clinical diagnosis. GN is defined by the presence of glomerular hematuria or albuminuria with or without an underlying systemic disease causing glomerulonephritis. The diagnosis of $\mathrm{DN}$ is based on albuminuria in a subject with type 2 diabetes mellitus and the presence of diabetic retinopathy. HTN is defined by the patient's hypertension history and the absence of a systemic illness associated with renal damage. Unified ultrasound criteria [13] will be used to diagnose PKD. The other causative diseases will be categorized as 'unclassified'.

\section{Data collection}

The detailed protocol for data and sample collection is described in Table 1. The eligible subjects will be screened and evaluated at baseline for socio-demographic information, smoking, alcohol consumption, medication, and detailed personal and family medical histories. The anthropometric measurements (height, weight, and waist/hip ratios), and measurements of the resting blood pressure (BP) in the clinic and pulse pressure using an electronic sphygmomanometer will be conducted. The subjects will complete questionnaires concerning quality of life, socioeconomic status, educational level, physical activity, health behaviors, and health care facility utilization. After enrollment in the study, the laboratory tests, cardiac evaluation and radiologic imaging will be performed according to the specific protocol. DNA, serum and urine samples will be initially collected. Serum and urine samples will be subsequently collected on a regular basis according to the standardized protocol. The details regarding the study visit and test schedule are shown in Table 1.

All of the data, including clinical information, laboratory results and outcome, will be entered into a web-based, electronic, case-reporting form (eCRF). The electronic data management system for the KNOW-CKD was developed by the division of data management in Seoul National University Medical Research Collaborating Center.

\section{Outcome variables}

A renal event is defined by a $>50 \%$ decrease in eGFR from the baseline values, doubling of serum creatinine, or ESRD. The estimated GFR for defining renal outcome will be based on the four-variable MDRD formula. However, the Chronic Kidney Disease Epidemiology Collaboration (CKD-EPI) creatinine equation [14] or the CKD-EPI creatinine-cystatin $C$ equation [15] will also be employed to estimate GFR.

At each visit, the subjects will be queried and evaluated for CV events, including myocardial infarction (fatal and nonfatal), coronary revascularization, stroke and new onset or aggravation of congestive heart failure. The causes of death will be verified and classified as cardiac, non-cardiac and unknown. An independent committee will adjudicate all of the primary outcomes. The subjects who withdraw from the study will be traced for this type of information with the help of the National Health Insurance System and Korea Statistical Information Service. The secondary outcomes are hospitalization, fracture, QOL, and economic status during the follow-up.

\section{Biospecimen collection}

Using an EDTA tube, $3 \mathrm{ml}$ of whole blood will be obtained and sent immediately for extracting DNA to the central laboratory. Another $10 \mathrm{ml}$ of whole blood will be obtained using the serum separation tube (SST) and centrifuged within 1 hour for serum separation and sent to the central laboratory for measurements of creatinine, cystatin $\mathrm{C}$, intact parathyroid hormone (iPTH), 25-hydroxyvitamine D3 (25D), 1,25-dihydroxyvitamin D3 (1,25D), and troponin T. Serum creatinine will be measured using an IDMS-traceable method; and cystatin $\mathrm{C}$ will be calculated using immunonephelometry calibrated against the reference material [15-17]. The first-voided urine samples $(15 \mathrm{ml})$ will be collected for assaying albumin, protein, calcium $(\mathrm{Ca})$, phosphorus $(\mathrm{P})$, urea, uric acid, electrolytes and osmolality at the central laboratory. The aliquots of the remaining serum and urine samples will be stored in a deep freezer $\left(-70^{\circ} \mathrm{C}\right)$ in two different places for future studies. Stringent rules for the sample harvest and processing will be applied to ensure accurate assays. Blood and urine sampling will be conducted under strict monitoring guidelines throughout the study period.

\section{Follow-up}

The participating subjects will visit the center according to the follow-up schedule. At each visit, the subjects will undergo scheduled tests; the investigators will review the subjects' recent medical histories and occurrence of events. We assumed that $10 \%$ of the individuals would withdraw from the study and that ESRD or death would develop subsequently in 
Table 1 Schedule of the KNOW-CKD at each visit

\begin{tabular}{|c|c|c|c|c|c|c|c|c|c|c|c|c|c|}
\hline \multirow{2}{*}{ Parameter } & \multicolumn{13}{|c|}{ Visit time (year) } \\
\hline & Screening & Baseline & 0.5 & 1 & 2 & 3 & 4 & 5 & 6 & 7 & 8 & 9 & 10 \\
\hline Informed consent & $\mathrm{O}$ & & & & & & & & & & & & \\
\hline Demographic information & $\mathrm{O}$ & & & & & & & & & & & & \\
\hline Medical History & $\mathrm{O}$ & & & & & & & & & & & & \\
\hline Eligibility confirmation & $\mathrm{O}$ & & & & & & & & & & & & \\
\hline Recent events & & & 0 & $\mathrm{O}$ & O & O & O & O & O & O & $\mathrm{O}$ & $\mathrm{O}$ & O \\
\hline Medications & & $\mathrm{O}$ & 0 & O & O & O & O & O & O & $\mathrm{O}$ & O & $\mathrm{O}$ & O \\
\hline Health Questionnaires & & $\mathrm{O}$ & & & & & & O & & & & & \\
\hline KDQOL questionnaires & & $\mathrm{O}$ & & & & & & $\mathrm{O}$ & & & & & \\
\hline Blood pressure & & $\mathrm{O}$ & 0 & $\mathrm{O}$ & O & O & O & $\mathrm{O}$ & O & $\mathrm{O}$ & $\mathrm{O}$ & $\mathrm{O}$ & $\mathrm{O}$ \\
\hline Anthropometry & & $\mathrm{O}$ & 0 & $\mathrm{O}$ & $\mathrm{O}$ & $\mathrm{O}$ & $\mathrm{O}$ & $\mathrm{O}$ & O & $\mathrm{O}$ & $\mathrm{O}$ & $\mathrm{O}$ & $\mathrm{O}$ \\
\hline $\mathrm{CBC}$, chemistry & & $\mathrm{O}$ & 0 & $\mathrm{O}$ & O & O & O & O & O & $\mathrm{O}$ & $\mathrm{O}$ & $\mathrm{O}$ & $\mathrm{O}$ \\
\hline IDMS-traceable Cr, MDRD eGFR & & O & 0 & $\mathrm{O}$ & O & O & O & $\mathrm{O}$ & O & $\mathrm{O}$ & $\mathrm{O}$ & $\mathrm{O}$ & $\mathrm{O}$ \\
\hline Cystatin C & & $\mathrm{O}$ & 0 & O & O & O & $\mathrm{O}$ & $\mathrm{O}$ & O & $\mathrm{O}$ & O & $\mathrm{O}$ & $\mathrm{O}$ \\
\hline HbA1c (diabetic subjects) & & $\mathrm{O}$ & 0 & $\mathrm{O}$ & O & O & O & O & O & $\mathrm{O}$ & $\mathrm{O}$ & $\mathrm{O}$ & $\mathrm{O}$ \\
\hline Lipid panel, CRP, iron profile & & $\mathrm{O}$ & & O & & O & & O & & $\mathrm{O}$ & & 0 & \\
\hline Intact PTH, 25D, 1,25 D, cardiac troponin T & & O & & O & & O & & O & & O & & $\mathrm{O}$ & \\
\hline Urinalysis & & O & 0 & O & O & O & O & $\mathrm{O}$ & O & O & O & $\mathrm{O}$ & $\mathrm{O}$ \\
\hline 24-h urine collection & & $\mathrm{O}$ & & & & O & & $\mathrm{O}$ & & $\mathrm{O}$ & & $\mathrm{O}$ & \\
\hline Electrolyte/Cr/osmolality, albumin/protein (Spot urine) & & O & & O & & O & & O & & O & & O & \\
\hline Electrocardiogram, chest $X$ ray & & O & & & O & & O & & O & & O & & O \\
\hline $\begin{array}{l}\text { Echocardiography, Pulse wave velocity, Ankle-brachial index, MDCT, } \\
\text { LS spine lateral X-ray for vascular calcification }\end{array}$ & & $\mathrm{O}$ & & & & & O & & & & $\mathrm{O}$ & & \\
\hline Bone mineral density & & $\mathrm{O}$ & & & & & O & & & & O & & $\mathrm{O}$ \\
\hline DNA sample & & $\mathrm{O}$ & & & & & & & & & & & \\
\hline Plasma/urine sample & & $\mathrm{O}$ & 0 & $\mathrm{O}$ & O & $\mathrm{O}$ & O & $\mathrm{O}$ & O & $\mathrm{O}$ & $\mathrm{O}$ & $\mathrm{O}$ & $\mathrm{O}$ \\
\hline Fundus exam (diabetic subjects) & & $\mathrm{O}$ & & & & & & & & & & & \\
\hline Abdomen CT or MRI (polycystic kidney disease) & & $\mathrm{O}$ & & & O & & O & & O & & $\mathrm{O}$ & & O \\
\hline Family screen (polycystic kidney disease) & & $\mathrm{O}$ & & & & & & & & & & & \\
\hline
\end{tabular}

CBC, complete blood count; KDQOL, Kidney Disease -Quality of Life; IDMS-serum cr, serum creatinine measurement using IDMS-traceable method; MDCT, multidetector computed tomography for coronary calcium score.

$10 \%$ of the subjects annually [18]. Efforts will be made to prevent drop-outs, i.e., providing free medical examinations, frequent telephone calls and dietary education.

\section{Institutional review board (IRB) approval}

The study protocol was approved by the IRB at each participating clinical center in 2011.

\section{Statistical power estimation}

With an overall sample size of 600 subjects, which is the size of the each subgroup (GN, DN, HTN, and PKD), $80 \%$ power is achieved to detect hazard ratios of approximately 2.00 and 1.60 for an exposure with prevalence of 0.1 and 0.5 and a dropout rate of $10 \%$.

\section{Statistical methods and plan}

The baseline characteristics of the study participants for each subgroup were presented with descriptive statistics using a one-way analysis of variance or Kruskal-Wallis test according to the distribution of continuous variables and a chi-square test or Fisher's exact test for categorical variables.

To analyze the association of outcome variables, the time-to-event analysis will be used to evaluate primary outcomes, such as renal events (initiation of renal replacement therapy and 50\% decline of GFR), CV events (myocardial infarction and ischemic stroke) and death. The KaplanMeier curves and the proportional hazard models will serve as major statistical methods for the outcomes. The incidence or mortality rates according to each outcome variable will be calculated for each etiologic group; the 
incidence ratios will be presented with reference to the diabetic nephropathy subgroup. The effect of exposure on the development of outcomes will be analyzed separately in each group and in the overall groups. The heterogeneity of each relative risk within the KNOW-CKD subcohort will be analyzed using the Cochran Q test or using a shared- frailty model, where time-dependent confounders should be adjusted [19]. The CKD progression will be evaluated using decreased GFR calculations. Because it is common for a patient with CKD to have a non-linear decline in GFR or a prolonged period of non-progression [20], we will assess the longitudinal trajectory of GFR for the individual

Table 2 General characteristics of study subjects participating in KNOW-CKD from 2011 to September, 2013

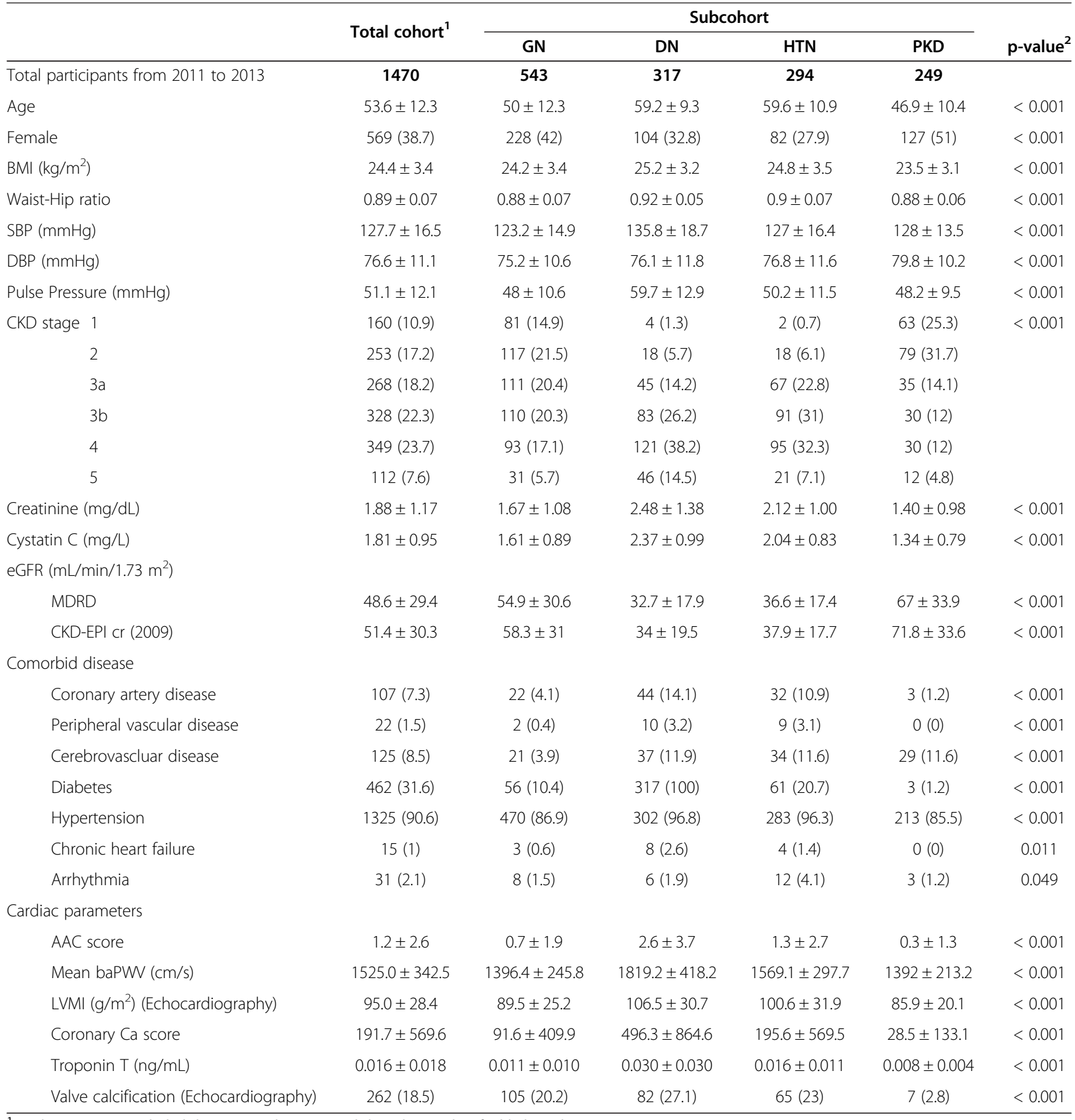

${ }^{1}$ Total participants included those in 4 subgroups and the other unclassified kidney diseases.

ANOVA or Kruskal-Wallis test for continuous variables and Chi-square or Fisher's Exact test for categorical variables.

Numbers denote mean \pm s.d. or number (\%).

Abbreviations: GN, glomerulonephropathy; DN , diabetic nephropathy; HTN, hypertensive nephropathy; PKD, autosomal dominant polycystic

kidney disease; AAC, abdominal aorta calcification; baPWV, brachial-to-ankle pulse wave velocity; LVMI, left ventricular mass index. 
Table 3 General characteristics of study participants according to CKD stage, KNOW-CKD from 2011 to September, 2013

\begin{tabular}{|c|c|c|c|c|c|c|c|}
\hline \multicolumn{8}{|c|}{ CKD Stage $^{1}$} \\
\hline & Stage 1 & Stage 2 & Stage $3 a$ & Stage $3 b$ & Stage 4 & Stage 5 & $\mathrm{p}$-value $^{2}$ \\
\hline Total participants from 2011 to 2013 & 160 & 253 & 269 & 328 & 350 & 112 & \\
\hline Age & $42.2 \pm 11.8$ & $49.6 \pm 11.9$ & $54.8 \pm 11.7$ & $56.7 \pm 10.7$ & $57.5 \pm 11$ & $54.5 \pm 11.6$ & $<0.001$ \\
\hline Female & $82(51.3)$ & $92(36.4)$ & $79(29.4)$ & $126(38.4)$ & $133(38)$ & $57(50.9)$ & $<0.001$ \\
\hline Height (cm) & $165.4 \pm 8.0$ & $166.3 \pm 8.5$ & $165.3 \pm 8.2$ & $163.5 \pm 8.1$ & $164.1 \pm 8.3$ & $162.0 \pm 8.8$ & $<0.001$ \\
\hline Weight (kg) & $64.9 \pm 12.8$ & $68.0 \pm 12.5$ & $67.6 \pm 11.5$ & $66.3 \pm 10.5$ & $65.8 \pm 11.4$ & $63.6 \pm 12.1$ & 0.005 \\
\hline BMI $\left(\mathrm{kg} / \mathrm{m}^{2}\right)$ & $23.6 \pm 3.7$ & $24.5 \pm 3.5$ & $24.7 \pm 3.3$ & $24.8 \pm 3.2$ & $24.4 \pm 3.3$ & $24.2 \pm 3.5$ & 0.001 \\
\hline Waist-Hip ratio & $0.87 \pm 0.06$ & $0.88 \pm 0.07$ & $0.89 \pm 0.07$ & $0.91 \pm 0.07$ & $0.91 \pm 0.06$ & $0.89 \pm 0.06$ & $<0.001$ \\
\hline SBP $(m m H g)$ & $126.3 \pm 15.6$ & $126.1 \pm 16.1$ & $126.3 \pm 15.3$ & $126.1 \pm 15.3$ & $129.4 \pm 17.3$ & $136.1 \pm 19.8$ & $<0.001$ \\
\hline $\mathrm{DBP}(\mathrm{mmHg})$ & $77.6 \pm 10.9$ & $77.2 \pm 11.9$ & $76.6 \pm 10.1$ & $75.5 \pm 10.4$ & $76.1 \pm 11.7$ & $78.7 \pm 11.8$ & 0.275 \\
\hline Pulse Pressure (mmHg) & $48.7 \pm 10$ & $48.9 \pm 10.3$ & $49.7 \pm 11.8$ & $50.5 \pm 12.3$ & $53.3 \pm 12.5$ & $57.4 \pm 14.2$ & $<0.001$ \\
\hline Urine ACR (mg/g cr) & & & & & & & $<0.001$ \\
\hline $\mathrm{A} 1(<30)$ & $53(34.4)$ & 71 (29.6) & $41(16.3)$ & $37(12.1)$ & $15(4.5)$ & $2(1.9)$ & \\
\hline A2 $(30 \sim 300)$ & $47(30.5)$ & $76(31.7)$ & $86(34.3)$ & $110(35.8)$ & $98(29.5)$ & $20(18.5)$ & \\
\hline A3 $(300 \leq)$ & $54(35.1)$ & $93(38.7)$ & $124(49.4)$ & $160(52.1)$ & $219(66)$ & $86(79.6)$ & \\
\hline Primary kidney disease & & & & & & & $<0.001$ \\
\hline GN & $81(51)$ & $117(46)$ & $111(41)$ & $110(34)$ & $93(27)$ & $31(28)$ & \\
\hline DN & $4(3)$ & $18(7)$ & $45(17)$ & $83(25)$ & $121(35)$ & $46(41)$ & \\
\hline HTN & $2(1)$ & $18(7)$ & $67(25)$ & $91(28)$ & $95(27)$ & $21(19)$ & \\
\hline PKD & $63(39)$ & $79(31)$ & $35(13)$ & $30(9)$ & $30(9)$ & $12(11)$ & \\
\hline Unclassified & $10(6)$ & $21(8)$ & $11(4)$ & $14(4)$ & $11(3)$ & $2(2)$ & \\
\hline \multicolumn{8}{|l|}{ Comorbid disease } \\
\hline Coronary artery disease & $4(2.5)$ & $7(2.8)$ & $24(9)$ & $30(9.2)$ & $36(10.4)$ & $6(5.4)$ & $<0.001$ \\
\hline Peripheral vascular disease & $1(0.6)$ & $2(0.8)$ & $4(1.5)$ & $1(0.3)$ & $13(3.7)$ & $1(0.9)$ & 0.010 \\
\hline Cerebrovascluar disease & $4(2.5)$ & $13(5.2)$ & $29(10.9)$ & $30(9.2)$ & $40(11.5)$ & $9(8.1)$ & 0.004 \\
\hline Diabetes & 19 (11.9) & $46(18.3)$ & $73(27.3)$ & $118(36.3)$ & $156(45)$ & $50(45)$ & $<0.001$ \\
\hline Hypertension & $126(78.8)$ & $217(86.1)$ & $241(90.3)$ & 305 (93.8) & $331(95.4)$ & 105 (94.6) & $<0.001$ \\
\hline Congestive heart failure & $0(0)$ & $0(0)$ & $1(0.4)$ & $3(0.9)$ & $10(2.9)$ & $1(0.9)$ & 0.007 \\
\hline Arrhythmia & $1(0.6)$ & $5(2)$ & $8(3)$ & $8(2.5)$ & $7(2)$ & $2(1.8)$ & 0.701 \\
\hline Creatinine (mg/dL) & $0.71 \pm 0.14$ & $1.00 \pm 0.18$ & $1.33 \pm 0.18$ & $1.75 \pm 0.27$ & $2.68 \pm 0.61$ & $4.75 \pm 1.36$ & $<0.001$ \\
\hline Cystatin C (mg/L) & $0.76 \pm 0.15$ & $1.00 \pm 0.20$ & $1.33 \pm 0.24$ & $1.76 \pm 0.33$ & $2.60 \pm 0.52$ & $3.89 \pm 0.70$ & $<0.001$ \\
\hline \multicolumn{8}{|l|}{ eGFR (mL/min/1.73 m²) } \\
\hline MDRD & $109.4 \pm 20.2$ & $73 \pm 8.6$ & $52.1 \pm 4.4$ & $37 \pm 4.2$ & $23.2 \pm 4.3$ & $11.8 \pm 2.3$ & $<0.001$ \\
\hline CKD-EPI cr (2009) & $110.9 \pm 10.4$ & $80.7 \pm 10.9$ & $55.8 \pm 5.5$ & $38.9 \pm 4.8$ & $23.8 \pm 4.7$ & $11.9 \pm 2.5$ & $<0.001$ \\
\hline Hemoglobin (g/dL) & $14 \pm 1.4$ & $13.9 \pm 1.7$ & $13.5 \pm 1.8$ & $12.8 \pm 1.8$ & $11.7 \pm 1.5$ & $10.6 \pm 1.2$ & $<0.001$ \\
\hline$P(\mathrm{mg} / \mathrm{dL})$ & $3.5 \pm 0.6$ & $3.5 \pm 0.6$ & $3.5 \pm 0.5$ & $3.6 \pm 0.6$ & $3.9 \pm 0.6$ & $4.7 \pm 0.7$ & $<0.001$ \\
\hline hsCRP (mg/L) & $1 \pm 2.3$ & $1.8 \pm 3.9$ & $2.2 \pm 6.4$ & $1.7 \pm 3$ & $3.1 \pm 8.1$ & $1.9 \pm 3.8$ & $<0.001$ \\
\hline Intact PTH (pg/mL) & $40.2 \pm 17.7$ & $38.8 \pm 14.2$ & $53.2 \pm 30.2$ & $55.7 \pm 21$ & $92.4 \pm 41.8$ & $220 \pm 118.3$ & $<0.001$ \\
\hline 25D (ng/mL) & $15.8 \pm 4.8$ & $18.9 \pm 6.7$ & $19.3 \pm 7.7$ & $18.7 \pm 7.3$ & $18.2 \pm 8.2$ & $17.1 \pm 7.8$ & $<0.001$ \\
\hline $1,25 \mathrm{D}(\mathrm{pg} / \mathrm{mL})$ & $30.5 \pm 7.6$ & $30.8 \pm 7.3$ & $28.5 \pm 7.5$ & $26.9 \pm 5.8$ & $23.5 \pm 5.3$ & $24.7 \pm 7.4$ & $<0.001$ \\
\hline \multicolumn{8}{|l|}{ Cardiac parameters } \\
\hline Mean baPWV (cm/s) & $1313.4 \pm 189.3$ & $1419.4 \pm 239.4$ & $1502 \pm 303.6$ & $1587 \pm 329.4$ & $1637 \pm 417.6$ & $1629.8 \pm 369.2$ & $<0.001$ \\
\hline LVMI (g/m²) (Echocardiography) & $85.5 \pm 23$ & $86.2 \pm 23.1$ & $89.9 \pm 23.9$ & $96.1 \pm 25.2$ & $104.1 \pm 31.2$ & $109.6 \pm 39.6$ & $<0.001$ \\
\hline
\end{tabular}


Table 3 General characteristics of study participants according to CKD stage, KNOW-CKD from 2011 to September, 2013 (Continued)

\begin{tabular}{lccccccc}
\hline Coronary Ca score & $44.2 \pm 225.7$ & $101.1 \pm 313.1$ & $230.7 \pm 705.3$ & $219.5 \pm 599.1$ & $252.9 \pm 634.9$ & $241.9 \pm 644.6$ & $<0.001$ \\
Troponin T (ng/mL) & $0.006 \pm 0.003$ & $0.008 \pm 0.004$ & $0.011 \pm 0.006$ & $0.016 \pm 0.015$ & $0.024 \pm 0.024$ & $0.031 \pm 0.030$ & $<0.001$ \\
Valve calcification (Echocardiography) & $12(7.6)$ & $31(12.6)$ & $39(15.3)$ & $75(23.5)$ & $75(22.5)$ & $30(28)$ & $<0.001$ \\
\hline
\end{tabular}

${ }^{1}$ CKD stage classified by MDRD equation.

ANOVA or Kruskal-Wallis test for continuous variables and Chi-square or Fisher's Exact test for categorical variables.

Numbers denote mean \pm s.d. or number (\%).

Abbreviations: GN, glomerulonephropathy; DN, diabetic nephropathy; HTN, hypertensive nephropathy; PKD, autosomal dominant polycystic kidney disease;

baPWV, brachial-to-ankle pulse wave velocity; LVMI, left ventricular mass index.

participants of KNOW-CKD using the Bayesian smoothing technique [21]. If the progression of CKD is steady and linear, the generalized estimating equation will be adopted to compare the slope of the GFR with repeated measurements. The progression of CKD will be supplemented with an event-based analysis, whereby the occurrence of ESRD or the substantial decrease of GFR will be treated as the events of interest.

\section{Results on interim analysis}

From July 2011 to September 2013, we enrolled 1,470 participants, including 543 GN, 317 DN, 294 HTN, 249 PKD sub-participants and remaining participants with unclassified subtypes. The baseline demographics and the laboratory values of KNOW-CKD are summarized in Tables 2 and 3. The mean age of the total CKD cohort at the time of study enrollment is $53.6 \pm 12.3$ years, $38.7 \%$ are female, and $31.6 \%$ diabetic. The proportion of each CKD stage is as follows; $10.9 \%$ (stage 1), $17.2 \%$ (stage 2), 18.2\% (stage 3a), $22.3 \%$ (stage $3 \mathrm{~b}$ ), $23.7 \%$ (stage 4 ) and $7.6 \%$ (stage 5 , nondialyzed). GN, DN, HTN and PKD were the most common causative diseases in descending order. At baseline, 7.3\% of the cohort had a history of coronary heart disease, $8.5 \%$ had cerebrovascular disease, and $90.6 \%$ had hypertension. In terms of the Charlson comorbidity score and various cardiac parameters, the DN subgroup exhibited the worst profiles (Figure 1 and Table 2). In subjects with advanced CKD stages, the elevated levels of pulse pressure, serum phosphorus, and intact PTH were more pronounced than the subjects with early stages. Hemoglobin levels, 25D and $1,25 \mathrm{D}$, were significantly lower and the left ventricular mass index (LVMI), coronary calcium score and valve calcification were higher in subjects with advanced CKD stages.

\section{Discussion}

The prevalence of CKD is increasing, as are the risk factors for CKD, such as diabetes, metabolic syndrome, and hypertension [22]. The National Health and Nutrition Examination Survey (NHANES) data from the U.S.A. show that the prevalence of CKD increased from $10 \%$ in the 19941998 cohort up to $13.1 \%$ in the 1999-2004 cohort [23].

CKD is a major risk factor for the development of cardiovascular complications and all-cause mortality. One large observational study reported that the risk of death increased

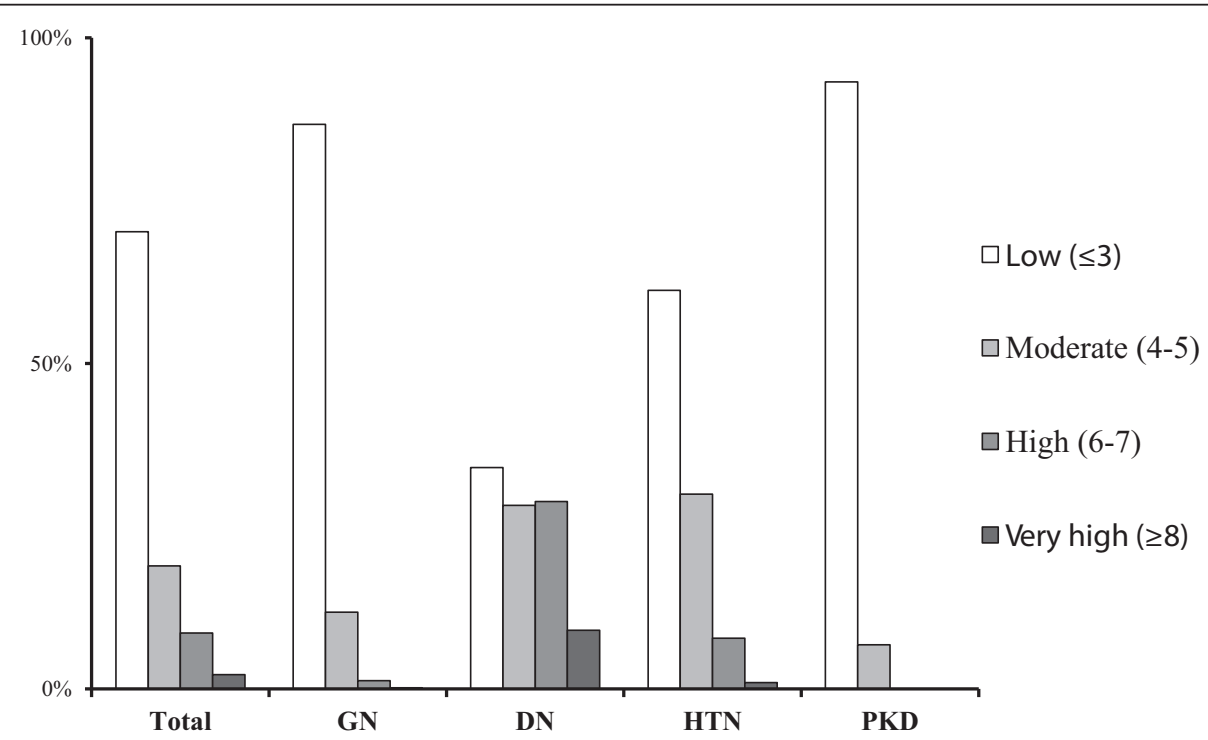

Figure 1 Age-adjusted modified Charlson comorbidity index in total and subgroup participants, KNOW-CKD 2011-2013. Abbreviations: GN, glomerulonephritis; DN, diabetic nephropathy; HTN, hypertensive nephropathy; PKD, polycystic kidney disease. 
dramatically as the GFR decreased below $60 \mathrm{ml} / \mathrm{min}$ per $1.73 \mathrm{~m}^{2}$ [3]. The adjusted hazard ratio for death was 3.2; the eGFR ranged from $15 \mathrm{ml} / \mathrm{min}$ to $29 \mathrm{ml} / \mathrm{min}$ per $1.73 \mathrm{~m}^{2}$.

In a recent meta-analysis of the general population cohort, including a total of more than one million individuals [24], the risk of death was fairly constant at eGFR of $75-105 \mathrm{ml} / \mathrm{min}$ per $1.73 \mathrm{~m}^{2}$. However, albuminuria, which is the earliest marker of glomerular damage, was independently associated with increased mortality, even in the presence of normal GFR. This point emphasizes the importance of albuminuria as a prognostic indicator, independent of GFR stages. Therefore, unlike previous CKD cohort studies, the KNOW-CKD included subjects with albuminuria or other early markers of kidney damage and with normal or mildly decreased GFR (CKD stage 1 to 2), as well as subjects with GFR $<60 \mathrm{ml} / \mathrm{min}$ per $1.73 \mathrm{~m}^{2}$ (CKD stages 3 to 5 ). The KNOW-CKD will also analyze various outcomes of CKD based on the specific causes of CKD and based on the GFR and albuminuria categories.

Many studies have shown that racial/ethnic disparities exist in mortality and cardiovascular outcome among individuals with CKD [25-28]. Asian patients with CKD generally have a lower burden of CV disease compared with other racial groups. The KNOW-CKD, which is the first large-scale cohort study with Korean patients with CKD, aims to explore the course of renal progression and $\mathrm{CV}$ events and to elucidate the risk factors for renal or CV outcomes in Korean subjects with CKD.

The original MDRD Study equation [29], which was developed in 1999, was modified for creatinine assays traceable to the international reference material for creatinine [12]. However, the accuracy and the precision of the MDRD equation have been controversial issues, especially at higher GFR. The CKD-EPI equation, which uses the same four variables as the MDRD Study equation, has less bias than the MDRD Study equation [14], especially at GFR $>60 \mathrm{ml} /$ min per $1.73 \mathrm{~m}^{2}$, and is suggested to be more accurate than the MDRD formula in Asian populations. The KNOWCKD will employ the MDRD Study equation [12], as well as the CKD-EPI creatinine [14] and the CKD-EPI creatinine-cystatin $C$ equations [15], to ensure the accuracy of the GFR estimation. Creatinine and cystatin $C$ assays will be conducted in a central laboratory, using assay methods traceable to the international reference materials $[16,17]$.

The KNOW-CKD will evaluate the complication profiles of anemia and mineral-metabolic derangements across the CKD stages. The KNOW-CKD will compare the clinical manifestations and natural course of the following four major renal etiologic subgroups: GN, DN, HTN, and PKD. Various biomarkers from the serum and urine samples will be evaluated to determine the risk prediction of adverse consequences. The genetic and epigenetic factors will be targeted. The KNOW-CKD will also evaluate the socio-economic burden and its impact on the quality of life, and the hospitalization of patients with CKD. Comparing the KNOW-CKD with other large-scale CKD cohorts through international collaboration may provide a platform for future research.

\section{Conclusion}

As the first large-scale CKD cohort study to be established and maintained longitudinally for up to 10 years, the KNOW-CKD will help to clarify the natural course, complication profiles, and risk factors of Asian populations with CKD. Including subjects in the early stages of CKD as well as those in the advanced stages and focusing on the causes will permit insight into CKD.

\section{Abbreviations}

GN: Glomerulonephropathy; DN: Diabetic nephropathy; HTN: Hypertensive nephropathy; PKD: Autosomal dominant polycystic kidney disease.

\section{Competing interests}

The authors declare that they have no conflicts of interest.

\section{Authors' contributions}

$\mathrm{K}-\mathrm{H} \mathrm{O}$ and SKP participated in the design of the study, reviewed and collected data using e-CRF and electronic medical records system, performed the statistical analysis, and drafted the manuscript. HCP participated in the analysis and interpretation of data. HJC, DWC, KHC, SHH, THY, KL, Y-SK, WC, Y-HH, SWK, YHK, and SWK participated in the design of the study, patient enrollment, acquisition of data, analysis, and interpretation of data. BJP and JL participated in the analysis and interpretation of data. CA had substantial contributions to funding, conception and design of the study, and draft and revision of the manuscript. All authors read and approved the final manuscript.

\section{Acknowledgment}

Authors thank the clinical research coordinators of each participating centers for their dedication in patient recruitment and data acquisition. Authors also thank the Medical Research Collaborating Center (MRCC), Seoul National University Hospital for their data management and statistical advice.

\section{Funding}

The KNOW-CKD was funded by grants 2011E3300300, $2012 \mathrm{E} 3301100$ and 2013 E3301600 from Research of Korea Centers for Disease Control and Prevention.

\section{KNOW-CKD: Study Group}

Clinical Centers. Seoul National University, Curie Ahn, MD (PI), Kook-Hwan Oh, MD, Dong Wan Chae, MD, Ho Jun Chin, MD, Hayne Cho Park, MD, Seungmi Lee, RN, Hyun Hwa Jang, RN and Hyun Jin Cho, RN. Yonsei University, Severance Hospital, Kyu Hun Choi, MD, Seung Hyeok Han, MD, Tae Hyun Yoo, MD and Mi Hyun Yu, RN. Kangbuk Samsung Medical Center, Kyubeck Lee, MD and Sooyeon Jin, RN. The Catholic University of Korea, Seoul St. Mary's Hospital, Yong-Soo Kim, MD and Sol Ji Kim, RN. Gachon University, Gil Hospital, Wookyung Chung, MD, Youkyoung Jang, RN and Ji Hye Park, RN. Eulji University, Eulji General Hospital. Young-Hwan Hwang, MD, Su-Ah Sung, $\mathrm{MD}$ and Jeong Ok So, RN. Chonnam University, Soo Wan Kim, MD and Ji Seon Lee. Inje University, Pusan Paik Hospital, Yeong Hoon Kim, MD, Sun Woo Kang, MD and Yun Jin Kim, RN.

Epidemiology and Biostatistics. Department of Preventive Medicine, Seoul National University College of Medicine, Byung-Joo Park, MD, Sue Kyung Park, MD and Juyeon Lee.

Coordinating Center. Medical Research Collaborating Center, Seoul National University Hospital and Seoul National University College of Medicine, Joongyub Lee, MD, Dayeon Nam, RN, Soohee Kang, MSc and Heejung Ahn, RN. Central Laboratory, Donghee Seo, MD, Lab Genomics, Korea and Dae Yeon Cho, PhD, Lab Genomics, Korea. Biobank. Korea Biobank, Korea Centers for Disease Control and Prevention, Osong, Korea. Korea Center for Disease Control and Prevention, Dukhyoung Lee, MD, Hyekyung Park, MD (Project Officer), Eunkyeong Jung (Project Officer), Suyeon Jeong, Eunmi Ahn and Sil-Hea Sung. 


\section{Author details}

'Department of Internal Medicine, Seoul National University, 101 Daehakro, Chongno Gu, Seoul 110-744, Korea. '2Department of Preventive Medicine, Seoul National University College of Medicine, Seoul, Korea. ${ }^{3}$ Department of Internal Medicine, Yonsei University, Severance Hospital, Seoul, Korea. ${ }^{4}$ Department of Internal Medicine, Kangbuk Samsung Medical Center, Sungkyunkwan University, Seoul, Korea. ${ }^{5}$ Department of Internal Medicine, The Catholic University of Korea, Seoul St. Mary's Hospital, Seoul, Korea. ${ }^{6}$ Department of Internal Medicine, Gachon University, Gil Hospital, Incheon, Korea. ${ }^{7}$ Department of Internal Medicine, Eulji University, Eulji General Hospital, Seoul, Korea. ${ }^{8}$ Department of Internal Medicine, Chonnam National University Medical School, Gwangju, Korea. ${ }^{9}$ Department of Internal Medicine, Inje University, Pusan Paik Hospital, Busan, Korea. ${ }^{10}$ Medical Research Collaborating Center, Seoul National University Hospital and Seoul National University College of Medicine, Seoul, Korea.

Received: 21 March 2014 Accepted: 7 May 2014

Published: 19 May 2014

\section{References}

1. Kim S, Lim CS, Han DC, Kim GS, Chin HJ, Kim SJ, Cho WY, Kim YH, Kim YS: The prevalence of chronic kidney disease (CKD) and the associated factors to CKD in urban Korea: a population-based cross-sectional epidemiologic study. J Korean Med Sci 2009, 24(Suppl):S11-21.

2. Anavekar NS, McMurray JJ, Velazquez EJ, Solomon SD, Kober L, Rouleau JL, White HD, Nordlander R, Maggioni A, Dickstein K, Zelenkofske S, Leimberger JD, Califf RM, Pfeffer MA: Relation between renal dysfunction and cardiovascular outcomes after myocardial infarction. N Engl J Med 2004, 351(13):1285-1295.

3. Go AS, Chertow GM, Fan D, McCulloch CE, Hsu CY: Chronic kidney disease and the risks of death, cardiovascular events, and hospitalization. $N$ Engl J Med 2004, 351(13):1296-1305

4. Astor BC, Matsushita K, Gansevoort RT, van der Velde M, Woodward M Levey AS, de Jong PE, Coresh J, El-Nahas M, Eckardt KU, Kasiske BL, Wright J, Appel L, Greene T, Levin A, Djurdjev O, Wheeler DC, Landray MJ, Townend JN, Emberson J, Clark LE, Macleod A, Marks A, Ali T, Fluck N, Prescott G, Smith DH, Weinstein JR, Johnson ES, Thorp ML, et al: Lower estimated glomerular filtration rate and higher albuminuria are associated with mortality and end-stage renal disease. A collaborative meta-analysis of kidney disease population cohorts. Kidney Int 2011, 79(12):1331-1340.

5. Gansevoort RT, Matsushita K, van der Velde M, Astor BC, Woodward M, Levey AS, de Jong PE, Coresh J: Lower estimated GFR and higher albuminuria are associated with adverse kidney outcomes. A collaborative meta-analysis of general and high-risk population cohorts. Kidney Int 2011, 80(1):93-104.

6. Levey AS, de Jong PE, Coresh J, El Nahas M, Astor BC, Matsushita K, Gansevoort RT, Kasiske BL, Eckardt KU: The definition, classification, and prognosis of chronic kidney disease: a KDIGO Controversies Conference report. Kidney Int 2011, 80(1):17-28.

7. Stevens PE, Levin A: Evaluation and management of chronic kidney disease: synopsis of the kidney disease: improving global outcomes 2012 clinical practice guideline. Ann Intern Med 2013, 158(11):825-830.

8. Feldman HI, Appel LJ, Chertow GM, Cifelli D, Cizman B, Daugirdas J, Fink JC, Franklin-Becker ED, Go AS, Hamm LL, He J, Hostetter T, Hsu CY, Jamerson K, Joffe M, Kusek JW, Landis JR, Lash JP, Miller ER, 3rd Mohler ER, Muntner P, Ojo AO, Rahman M, Townsend RR, Wright JT, Chronic Renal Insufficiency Cohort (CRIC) Study Investigators: The Chronic Renal Insufficiency Cohort (CRIC) Study: Design and Methods. J Am Soc Nephrol 2003, 14(7 Suppl 2):S148-153.

9. Lash JP, Go AS, Appel LJ, He J, Ojo A, Rahman M, Townsend RR, Xie D, Cifelli D, Cohan J, Fink JC, Fischer MJ, Gadegbeku C, Hamm LL, Kusek JW, Landis JR, Narva A, Robinson N, Teal V, Feldman HI, Chronic Renal Insufficiency Cohort (CRIC) Study Group: Chronic Renal Insufficiency Cohort (CRIC) Study: baseline characteristics and associations with kidney function. Clin J Am Soc Nephrol 2009, 4(8):1302-1311.

10. Imai E, Matsuo S, Makino H, Watanabe T, Akizawa T, Nitta K, limuro S, Ohashi Y, Hishida A: Chronic Kidney Disease Japan Cohort (CKD-JAC) study: design and methods. Hypertens Res 2008, 31(6):1101-1107.

11. Levin A, Rigatto C, Brendan B, Madore F, Muirhead N, Holmes D, Clase CM, Tang M, Djurdjev O, Can P: Cohort profile: Canadian study of prediction of death, dialysis and interim cardiovascular events (CanPREDDICT). BMC Nephrol 2013, 14:121.

12. Levey AS, Coresh J, Greene T, Stevens LA, Zhang YL, Hendriksen S, Kusek JW, Van Lente F: Using standardized serum creatinine values in the modification of diet in renal disease study equation for estimating glomerular filtration rate. Ann Intern Med 2006, 145(4):247-254.

13. Belibi FA, Edelstein CL: Unified ultrasonographic diagnostic criteria for polycystic kidney disease. J Am Soc Nephrol 2009, 20(1):6-8.

14. Levey AS, Stevens LA, Schmid CH, Zhang YL, Castro AF 3rd, Feldman HI, Kusek JW, Eggers P, Van Lente F, Greene T, Coresh J, CKD-EPI (Chronic Kidney Disease Epidemiology Collaboration): A new equation to estimate glomerular filtration rate. Ann Intern Med 2009, 150(9):604-612.

15. Inker LA, Schmid CH, Tighiouart H, Eckfeldt JH, Feldman HI, Greene T, Kusek JW, Manzi J, Van Lente F, Zhang YL, Coresh J, Levey AS, CKD-EPI Investigators: Estimating glomerular filtration rate from serum creatinine and cystatin C. N Engl J Med 2012, 367(1):20-29.

16. Grubb A, Blirup-Jensen S, Lindstrom V, Schmidt C, Althaus H, Zegers I: First certified reference material for cystatin $C$ in human serum ERM-DA471/ IFCC. Clin Chem Lab Med 2010, 48(11):1619-1621.

17. Blirup-Jensen S, Grubb A, Lindstrom V, Schmidt C, Althaus H: Standardization of Cystatin C: development of primary and secondary reference preparations. Scand J Clin Lab Invest Supp/ 2008, 241:67-70.

18. Hoefield RA, Kalra PA, Baker P, Lane B, New JP, O'Donoghue DJ, Foley RN, Middleton RJ: Factors associated with kidney disease progression and mortality in a referred CKD population. Am J Kidney Dis 2010, 56(6):1072-1081.

19. Ratcliffe SJ, Guo W, Ten Have TR: Joint modeling of longitudinal and survival data via a common frailty. Biometrics 2004, 60(4):892-899.

20. Li L, Astor BC, Lewis J, Hu B, Appel LJ, Lipkowitz MS, Toto RD, Wang X, Wright JT Jr, Greene TH: Longitudinal progression trajectory of GFR among patients with CKD. Am J Kidney Dis 2012, 59(4):504-512.

21. Crainiceanu CM, Ruppert D, Wand MP: Bayesian analysis for penalized spline regression using WinBUGS. J Stat Softw 2005, 14(14).

22. Nagata M, Ninomiya T, Doi $Y$, Yonemoto K, Kubo M, Hata J, Tsuruya K, lida $M$, Kiyohara $Y$ : Trends in the prevalence of chronic kidney disease and its risk factors in a general Japanese population: the Hisayama Study. Nephrol Dial Transplant 2010, 25(8):2557-2564.

23. Coresh J, Selvin E, Stevens LA, Manzi J, Kusek JW, Eggers P, Van Lente F, Levey AS: Prevalence of chronic kidney disease in the United States. JAMA 2007, 298(17):2038-2047.

24. Matsushita K, van der Velde M, Astor BC, Woodward M, Levey AS, de Jong PE, Coresh J, Gansevoort RT: Association of estimated glomerular filtration rate and albuminuria with all-cause and cardiovascular mortality in general population cohorts: a collaborative meta-analysis. Lancet 2010, 375(9731):2073-2081.

25. Mehrotra R, Kermah D, Fried L, Adler S, Norris K: Racial differences in mortality among those with CKD. J Am Soc Nephrol 2008, 19(7):1403-1410.

26. Muntner P, Newsome B, Kramer H, Peralta CA, Kim Y, Jacobs DR Jr, Kiefe Cl, Lewis CE: Racial differences in the incidence of chronic kidney disease. Clin J Am Soc Nephrol 2012, 7(1):101-107.

27. Mok CC: Racial difference in the prognosis of lupus nephritis. Nephrology (Carlton) 2010, 15(4):480-481.

28. Jovanovich A, Chonchol M, Cheung AK, Kaufman JS, Greene T, Roberts WL, Smits G, Kendrick J: Racial differences in markers of mineral metabolism in advanced chronic kidney disease. Clin J Am Soc Nephrol 2012, 7(4):640-647.

29. Levey AS, Bosch JP, Lewis JB, Greene T, Rogers N, Roth D: A more accurate method to estimate glomerular filtration rate from serum creatinine: a new prediction equation. Modification of Diet in Renal Disease Study Group. Ann Intern Med 1999, 130(6):461-470.

\section{doi:10.1186/1471-2369-15-80}

Cite this article as: Oh et al:: KNOW-CKD (KoreaN cohort study for Outcome in patients With Chronic Kidney Disease): design and methods. BMC Nephrology 2014 15:80. 